\title{
Baseline analysis of Mycoplasma mycoides subsp. mycoides antigens as targets for a DIVA assay for use with a subunit vaccine for contagious bovine pleuropneumonia
}

Harrison O. Lutta ${ }^{1 *}$ (D), David Odongo ${ }^{2}$, Arshad Mather ${ }^{3}$, Jose Perez-Casal ${ }^{4}$, Andrew Potter $^{4}$, Volker Gerdts $^{4}$, Emil M. Berberov ${ }^{4}$, Tracy Prysliak ${ }^{4}$, Martina Kyallo ${ }^{5}$, Alexander Kipronoh ${ }^{6}$, Moses Olum ${ }^{6}$, Roger Pelle ${ }^{5+}$ and Jan Naessens ${ }^{5+}$

\begin{abstract}
Background: Mycoplasma mycoides subsp. mycoides $(\mathrm{Mmm})$ is the causative agent of contagious bovine pleuropneumonia in cattle. A prototype subunit vaccine is being developed, however, there is currently no diagnostic test that can differentiate between infected cattle and those vaccinated with the prototype subunit vaccine. This study characterized $\mathrm{Mmm}$ proteins to identify potential antigens for use in differentiating infected from vaccinated animals.

Results: Ten Mmm antigens expressed as recombinant proteins were tested in an indirect ELISA using experimental sera from control groups, infected, and vaccinated animals. Data were imported into R software for analysis and drawing of the box and scatter plots while Cohen's Kappa assessed the level of agreement between the Mmm antigens. Two vaccine antigens (MSC_0499 and MSC_0776) were superior in detecting antibodies in sera of animals vaccinated with the subunit vaccines while two non-vaccine antigens (MSC_0636 and LppB) detected antibodies in sera of infected animals showing all clinical stages of the disease. Sensitivity and specificity of above $87.5 \%$ were achieved when the MSC_0499 and MSC_0636 antigens were tested on sera from vaccinated and infected animals.

Conclusions: The MSC_0499 and MSC_0776 antigens were the most promising for detecting vaccinated animals, while MSC_0636 and LppB were the best targets to identify infected animals. Further testing of sera from vaccinated and infected animals collected at different time intervals in the field should help establish how useful a diagnostic test based on a cocktail of these proteins would be.
\end{abstract}

Keywords: Antigens, Cattle, CBPP, DIVA, Mycoplasma mycoides

\footnotetext{
*Correspondence: holutta_006@yahoo.com

Roger Pelle and Jan Naessens share the last authorship

${ }^{1}$ Kenya Agricultural and Livestock Research Organization, Biotechnology

Research Institute, P.O. Box 14733-00800, Nairobi, Kenya

Full list of author information is available at the end of the article
}

(c) The Author(s). 2020 Open Access This article is licensed under a Creative Commons Attribution 4.0 International License, which permits use, sharing, adaptation, distribution and reproduction in any medium or format, as long as you give appropriate credit to the original author(s) and the source, provide a link to the Creative Commons licence, and indicate if changes were made. The images or other third party material in this article are included in the article's Creative Commons licence, unless indicated otherwise in a credit line to the material. If material is not included in the article's Creative Commons licence and your intended use is not permitted by statutory regulation or exceeds the permitted use, you will need to obtain permission directly from the copyright holder. To view a copy of this licence, visit http://creativecommons.org/licenses/by/4.0/. The Creative Commons Public Domain Dedication waiver (http://creativecommons.org/publicdomain/zero/1.0/) applies to the data made available in this article, unless otherwise stated in a credit line to the data. 


\section{Background}

Mycoplasma mycoides subsp. mycoides $(\mathrm{Mmm})$, the causative pathogen of contagious bovine pleuropneumonia (CBPP) in cattle, belongs to the classical ' $M y c o-$ plasma mycoides cluster' [1]. Clinically, CBPP manifests as either acute, sub-acute, or chronic forms. The acute and sub-acute forms are characterized by rapid breathing, fever, nasal discharge, cough, and sudden death; whereas the chronic stage of infection is characterized by weight loss and cough on exertion. During the acute phase of the disease, autonomous lung lesions and pleural fluid are often observed on post mortem examination [2]. CBPP financial losses and control costs are estimated at $€ 44.8$ million per annum in Africa [3]. To help combat the disease, two live attenuated vaccines namely T1/44 and T1/SR, are currently in use. A complement fixation test (CFT) and a competitive ELISA (cELISA) are the only prescribed World Organization for Animal Health (OIE) serological diagnostic tests to work with the T1/44 and T1/SR live-attenuated vaccines. The approved live attenuated vaccines and prescribed diagnostic tests have shortcomings, which necessitates the development of more effective vaccines and diagnostics.

To develop a vaccine and a supporting diagnostic that can differentiate CBPP-infected from vaccinated cattle (DIVA), we used a reverse vaccinology approach described in Perez-Casal et al., [4] to identify 28 potential $M m m$ vaccine antigens from the Gladysdale [5] and PG1 [6] genomes for a candidate subunit vaccine and a DIVA assay. Male Boran cattle in a study by Nkando et al., [7] were immunized using pools of five antigens previously identified [4], followed by a challenge with the Afadé $M m m$ strain. Two of the groups immunized with five proteins each showed protection after the $\mathrm{Mmm}$ challenge [7]. Moreover, seventeen immunogenic $\mathrm{Mmm}$ proteins evaluated in a cocktail indirect ELISA (iELISA) in a study by Heller et al., [8], displayed strong serological responses and high disease specificity as confirmed in earlier studies [9-13]. MSC_0136 and MSC_0636 showed sensitivity and specificity of above 85.6 and $96.4 \%$, respectively [8]. In 2018, screening of sera from naïve and CBPP-infected cattle using CFT and iELISA revealed that the iELISA based on the LppB $\mathrm{Mmm}$ antigen was more sensitive than CFT, which is considered a gold standard in the diagnosis of CBPP [14].

The development of subunit vaccines will allow the development of CBPP diagnostic tests that can differentiate infected from vaccinated animals. Sera from cattle immunized with a live attenuated vaccine could test positive for many $\mathrm{Mmm}$ antigens. Detection of antibodies against proteins not present in the subunit vaccine would discriminate between cattle immunized with a live-attenuated vaccine or exposed to $\mathrm{Mmm}$ in the field, and those vaccinated with a subunit vaccine containing known $\mathrm{Mmm}$ antigens. This study aimed to identify diagnostic antigens of $\mathrm{Mmm}$ capable of differentiating cattle infected with CBPP from those vaccinated with the subunit vaccines for the control of CBPP. The control of CBPP would reduce morbidity and mortality in cattle and tremendously improve the revenue of pastoral communities.

\section{Results}

\section{Selection of proteins for indirect ELISA}

Six $M m m$ proteins were evaluated as vaccine antigens in the prototype subunit vaccines, and four non-vaccine antigens selected based on their potential to detect infected cattle, as reported in previous studies were tested against sera from control groups, CBPP-infected and subunit vaccinated animals (Table 2).

\section{Characterization of sera from naive and CBPP infected cattle}

Sera from naïve and CBPP infected cattle were tested against the non-vaccine antigens. None of the animals seroconverted before infection with $\mathrm{Mmm}$ (Table 3). When non-vaccine antigens were used to detect antibodies in sera from animals challenged with $\mathrm{Mmm}$, MSC_0636 and LppB detected more animals as $\mathrm{Mmm}$ positive than MSC_0397 and MSC_0653. The iELISA results showed that MSC_0636 detected 6/8, 4/8, and 7/8 animals in acute, subacute, and chronic stages of the disease, respectively, while LppB detected $4 / 8,5 / 8$, and $7 / 8$ animals. However, MSC_0397 detected 3/8, 2/8, and 5/8 animals in acute, subacute, and the chronic stages of the disease, respectively, while MSC_0653 detected 3/8, 4/8 and $7 / 8$ animals. When non-vaccine antigens were tested on sera from subunit-vaccinated cattle to establish whether they could discriminate these from the CBPPinfected cattle, MSC_0653 detected antibodies in sera from vaccinated cattle, rendering it unsuitable for a DIVA diagnostic test. MSC_0636 showed a significance difference $(P<0.05)$ in discriminating infected from vaccinated animals, as did LppB (Additional files 1 and 2). Thus, these two antigens (MSC_636 and LppB) appear suitable for a DIVA diagnostic test.

\section{Characterization of sera from control group and subunit vaccinated cattle}

To define vaccine antigens that were targeted by antibody responses in naive and subunit vaccinated cattle, we determined the antibody responses against antigens in an experimental subunit vaccine. Antibodies were detected against four out of five antigens (MSC_0136, MSC_0431, MSC_0499, and MSC_0776) in the group C vaccine (Table 1 ) at or above the titer of 1:1600. None of the animals seroconverted before vaccination (Table 3). Figure 1 shows representative iELISA results using 
Table 1 Details of trial vaccines and control

\begin{tabular}{llll}
\hline Group & Antigen $(50 \mu \mathrm{g} /$ dose $)$ & Adjuvant & No. of cattle (n) \\
\hline A & None (Control group) & Montanide ISA61 VG & 8 \\
B & MSC_0136, MSC_0431, MSC_0499 and MSC_0775 & Montanide ISA61 VG & 8 \\
C & MSC_0136, MSC_0431, MSC_0499, MSC_0776 and MSC_0957 & Montanide ISA61 VG & 8 \\
\hline
\end{tabular}

MSC_0431 from sera from the control group and the group vaccinated with the test antigens. The trend shown in Fig. 1 with MSC_0431 was also observed using other vaccine antigens, MSC_0136, MSC_0499, and MSC_0776 in the subunit vaccine group $\bar{C}$ (data not shown). However, when vaccine antigens were tested on sera from CBPP-infected cattle to establish whether they could discriminate these from subunit-vaccinated cattle, MSC_0431 detected antibodies in sera from infected cattle, rendering it unsuitable for a DIVA diagnostic test. The results with MSC_0499 when used to test sera from vaccinated cattle, showed a significance difference $(P<$ $0.05)$ to those from infected animals, as did those using MSC_0776, thus making the two antigens suitable for a DIVA test (Additional files 1 and 2).

\section{Determination of cut-off point, diagnostic sensitivity, and} specificity of $\mathrm{Mmm}$ antigens

Serum samples from naïve, CBPP-infected and vaccinated cattle returned a mean \pm standard deviation OD values of $0.10 \pm 0.03$ (OD range of $0.07-0.19$ ), $0.23 \pm 0.14$ (OD range of $0.15-0.66$ ), and $0.24 \pm 0.20$ (OD range of $0.15-0.85)$, respectively. The change point for negative controls was as good as the cut-off point at an OD of $\leq 0.2$ at a wavelength of $405 \mathrm{~nm}$, at a titer of 1:1600 for vaccinated animals and 1:400 for infected cattle. There was a poor index value among the iELISA based on 10 $M m m$ antigens on sera from CBPP-infected, and subunit-vaccinated cattle, respectively (Cohen's Kappa statistic $\mathrm{k}=0.398 ; 0.239$, Table 2). The Cochran's $\mathrm{Q}$ test showed that the diagnostic sensitivity of MSC_0636, LppB, MSC_0499, and the MSC_0776 differed significantly from the rest of the antigens on sera from CBPPinfected and subunit-vaccinated cattle, respectively $(\mathrm{Q}=$ 41.013; $\mathrm{df}=9, p<0.001 ; \mathrm{Q}=54.419, \mathrm{df}=9, p<0.001)$.

For the vaccine antigens, there were no significant variations in medians of ODs between $\mathrm{Mmm}$ antigens on sera from the control/naive group (Fig. 2). However, there were significant variations in medians of ODs between $\mathrm{Mmm}$ antigens post-vaccination with the prototype recombinant vaccines. MSC_0499 showed a statistically significant ability to differentiate vaccinated from infected animals as did MSC_0776 (Figs. 3 and 4, Additional file 3).

When the non-vaccine antigens were assessed, MSC_ 0636 showed a statistically significant ability to differentiate infected from vaccinated animals, especially in the chronic clinical phase as did LppB (Figs. 3 and 5,

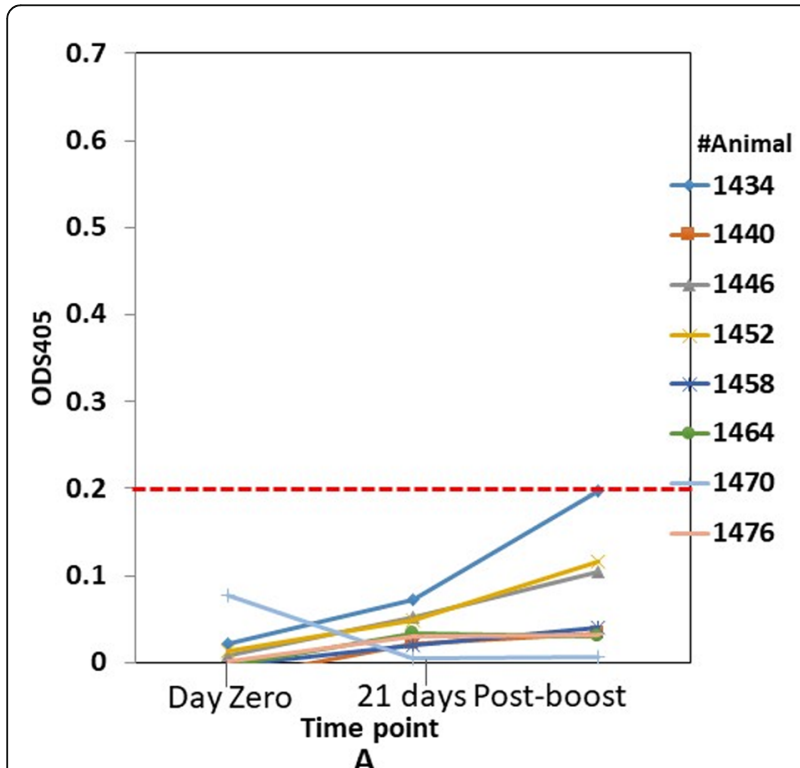

Fig. 1 Representative iELISA results of the control group (a), and those formulated with test antigens (b). The iELISA results were based on MSC_0431 Mmm antigen screened on sera post-boost (time point at second immunization). Seroconversion was observed in the group formulated with the test antigens but not the control group

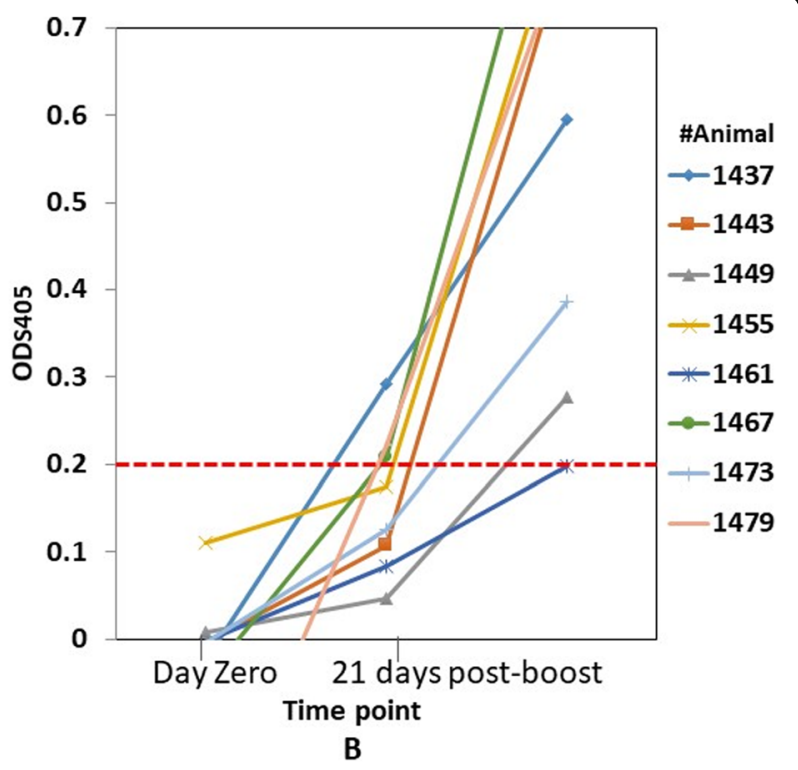


Table 2 Proteins used in this study

\begin{tabular}{llll}
\hline Name & Description & Size (kDal) & References \\
\hline \multicolumn{2}{c}{ Vaccine antigens $^{\text {a }}$} & & \\
MSC_0136 & Hypothetical lipoprotein & 66 & $11 ; 12$ \\
MSC_0431 & Prolipoprotein & 70 & $11 ; 12$ \\
MSC_0499 & Prolipoprotein & 111 & 4 \\
MSC_0775 & Prolipoprotein & 81 & 4 \\
MSC_0776 & Prolipoprotein & 120 & 4 \\
MSC_0957 & Prolipoprotein & 79 & 4 \\
Non-vaccine antigens & & \\
MSC_0397 & Prolipoprotein & 45 & $10 ; 11$ \\
MSC_0636 & Hypothetical lipoprotein & 50 & 12 \\
MSC_0653 & Prolipoprotein & 75 & 11 \\
LppB & Lipoprotein & 27 & 24 \\
\hline
\end{tabular}

${ }^{a}$ Vaccine antigens refer to proteins used to formulate prototype subunit vaccines

${ }^{b}$ Non-vaccine antigens refer to proteins selected based on their potential to detect CBPP-infected cattle as found in the literature

Additional file 4). MSC_0499, MSC_0776, MSC_0636, and $\mathrm{LppB}$ were therefore, determined to be the best antigens for a DIVA diagnostic, as they yielded specificities of $100 \%$ and sensitivities of between $62.5-100 \%$ (Table 3, Additional files 3 and 5).

\section{Discussion}

Recently, our laboratories developed subunit vaccines using $\mathrm{Mmm}$ recombinant antigens and demonstrated that they elicit protective immunity against experimental endotracheal challenge with virulent $\mathrm{Mmm}$ Afade strain [7]. In Phase 1, the study tested the protective effect of 14 pools of five recombinant antigens in a CBPP challenge, and three of these pools showed clear indications of a reduction in the pathological index [7]. In Phase II, different formulations of these recombinant vaccines were evaluated by their immune responses as described by Nkando et al., [7]. In this study, the $\mathrm{Mmm}$ antigens were used to analyze antibody responses in cattle infected with $\mathrm{Mmm}$ Afade strain, those vaccinated with the subunit vaccines, and naïve control groups to develop a DIVA diagnostic test.

Several studies describe the development of DIVA diagnostics/vaccines for M. bovis [15-17]. Zhang et al., [17] used the $M$. bovis-150 strain to develop the live attenuated vaccine that elicited protection against a challenge with the virulent strain of M. bovis HB0801. DIVA assays using sodium thiocyanate $(\mathrm{NaSCN})$ or recombinant proteins assay for use alongside the $M$. bovis-150 live attenuated vaccine have been reported $[15,16]$. Han et al. [15], used NaSCN in a competitive iELISA for the

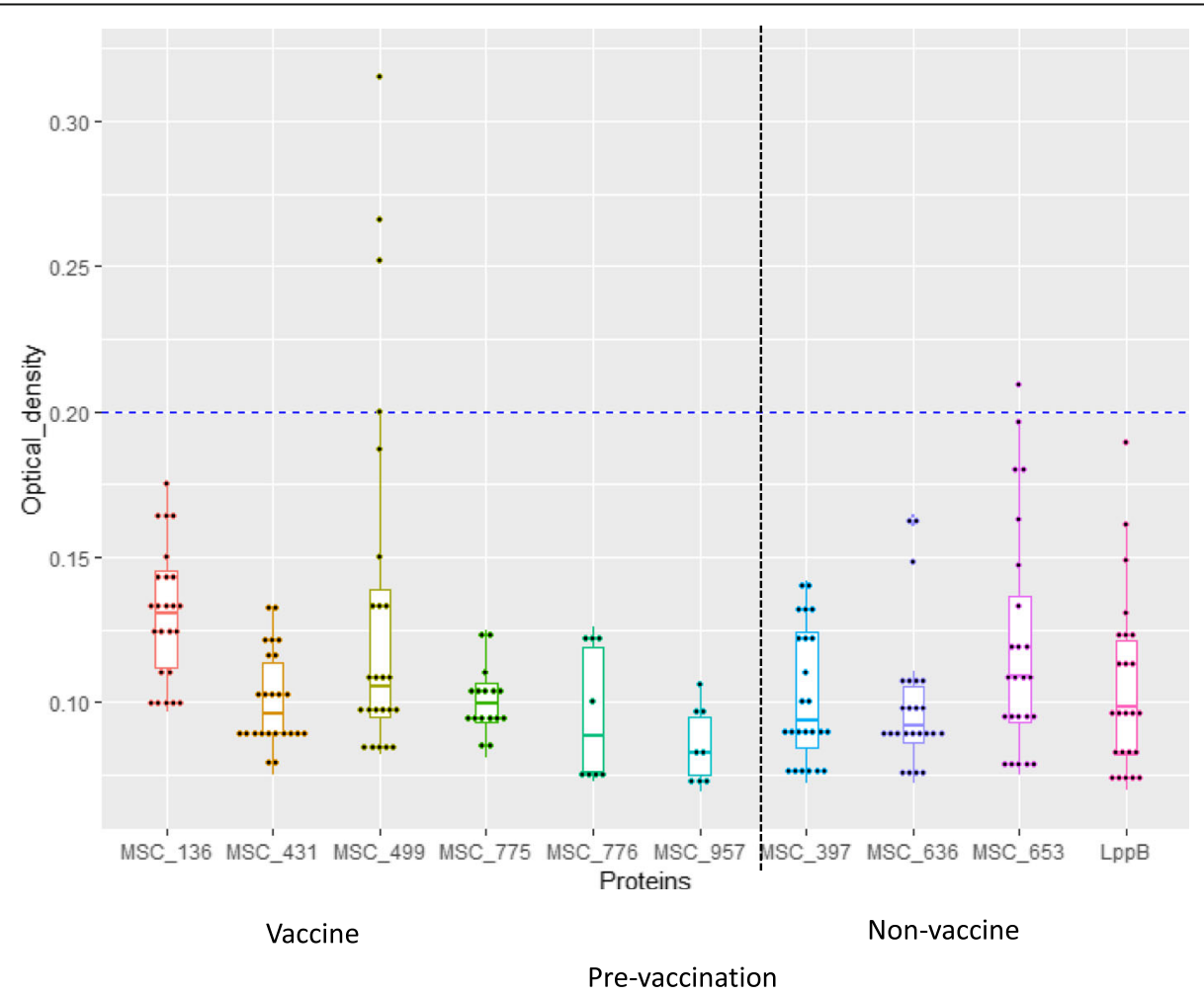

Fig. 2 Box and scatter plots showing ODs for different Mmm antigens screened against sera pre-vaccination. None of the antigens showed activity with sera from naïve, pre-vaccinated animals at an OD cut-off of 0.2 


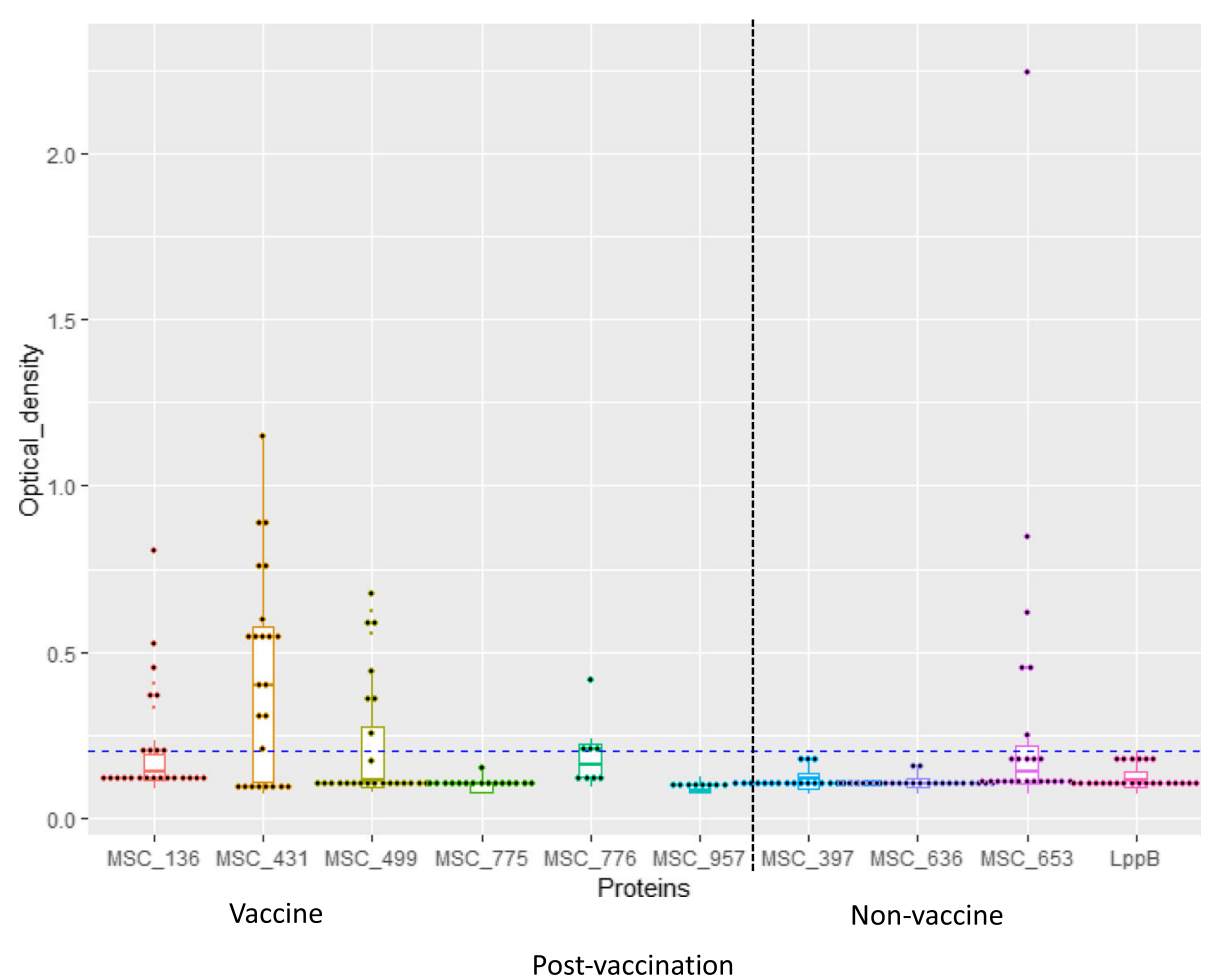

Fig. 3 Box and scatter plots representing ODs for Mmm antigens screened against sera from vaccinated cattle. The horizontal blue dotted line is at $\mathrm{OD}=0.2$. Although MSC_0653 was a non-vaccine antigen and not part of the prototype vaccines, the antigen detected antibodies in sera from vaccinated animals

detection of IgG. Khan et al., [16], used proteomic techniques to identify and characterize membrane-associated proteins of $M$. bovis HB0801 and its attenuated strain (M. bovis-150). The results identified a putative lipoprotein encoded by a functionally unknown gene MbovP730 that demonstrated high sensitivity and specificity in an IgG iELISA. A Western blot test confirmed that MbovP730 is absent in attenuated M. bovis-150, indicating that this antigen could be used to develop a DIVA assay.

In a study to establish a DIVA diagnostic test to be used in combination with the CBPP live-attenuated vaccine, a very immunogenic antigen, LppQ was characterized and an LppQ-knock-out derivative of the T1/44 live attenuated vaccine strain, the T1LppQ-MT1 was created [18-20]. It was suggested that LppQ could be used in a DIVA assay for discriminating animals vaccinated with the depleted T1LppQ-MT1 strain from those infected with the strains from the field [21, 22]. However, a genetically modified live attenuated vaccine might not be easily adopted by regulatory agencies, and there remains the disadvantages associated with a live attenuated vaccine including, the need for cold chain and possible side effects at the site of inoculation [23].

To develop a DIVA assay to be used with the newly developed CBPP subunit vaccines, four previously characterized antigens were used to test cattle sera to identify the most reliable antigen for identifying the infected and naïve animals. The four antigens (MSC_0397, MSC_0636, MSC_0653 and LppB) were selected based on extensive literature review and earlier laboratory work [4, 7, 8, 10, 12, 24]. Miltiadou et al., [24] characterized LppB and predicted that it could be a possible antigen for use in a serological diagnostic test. Our study supports this prediction based on the high sensitivity and specificity observed. Although Lutta et al., [14] showed that LppB detects chronically infected cattle, limited data were available to show that $\mathrm{LppB}$ could also detect the acute stages of CBPP.

Previously, Naseem et al., [12] obtained 100\% sensitivity and specificity in an ELISA test using both a conserved hypothetical (MSC_0636) and a glycosyltransferase (MSC_0108) $\mathrm{Mmm}$ proteins. The findings by Naseem et al., [12] are in the range of those obtained in this study, sensitivities of MSC_0636 and LppB being $87.5 \%$ with both antigens showing specificities of $100 \%$. The results by Naseem et al., [12] using MSC_0636 and those obtained in this study seem to reach the same conclusions as those of Heller et al., [8], who identified MSC_0136, MSC_0397, and MSC_0636 as the best-performing proteins in a cocktail iELISA. Heller at al., [8] obtained sensitivity and specificity 


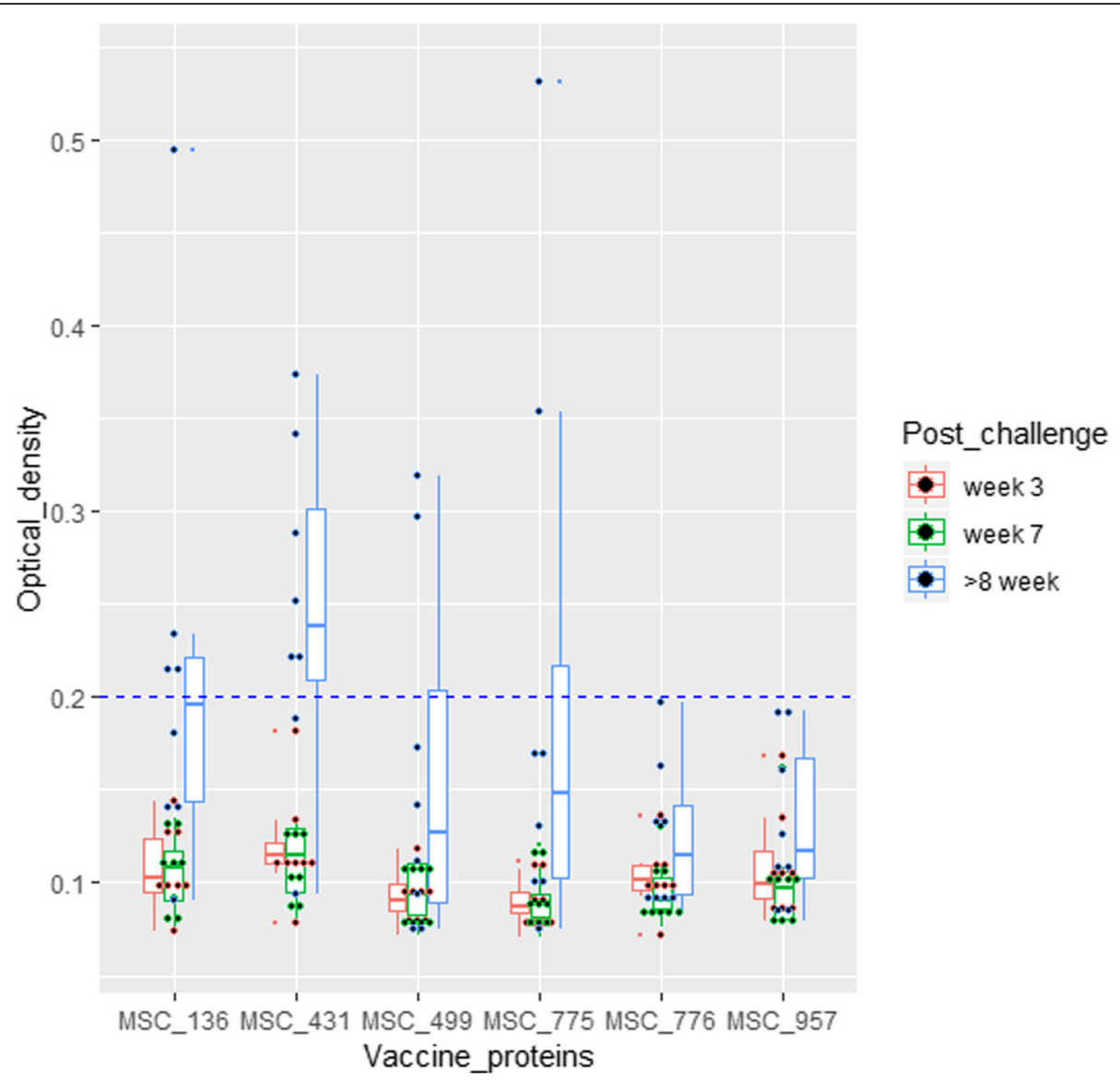

Fig. 4 Box and scatter plots showing ODs of vaccine antigens screened against sera from CBPP-infected cattle. Although MSC_0431 and MSC_0136 were part of the prototype vaccines, the antigens detected more antibodies in sera from chronically infected animals at an OD cut-off 0.2

values of above 85.6 and $96.4 \%$ respectively in a cocktail ELISA using MSC_0136 and MSC_0636, which were similar to those obtained in our study. Although Naseem et al., [12] reported sensitivity and specificity values of $100 \%$ using MSC_0108, the performance of this antigen was lower than MSC_0397 in the study by Heller et al., [8] using a large number of serum samples. Therefore, we did not include MSC_0108 in our study. The results of our study show that MSC_0636 and LppB detected antibodies in sera for both acute and chronic stages of CBPP. Although we chose a relatively high cut-off $\left(\mathrm{OD}_{405}\right.$ at $\left.\leq 0.2\right)$ in our iELISA assays to increase specificity, the sensitivity was not affected as earlier reported [25]. It was also noted that all animals seroconverted by day 142 .

We did not compare the same cattle sera used by previous authors and therefore, before the final validation of the DIVA diagnostic test proposed in our study, more cattle sera from field trials and from different time points after infection should be tested. A promising result is that the sensitivities and specificities obtained in this study are comparable to those of CFT and c-ELISA as prescribed by the OIE for the serological diagnosis of CBPP [26]. It was also noted that all antigens used in this study detected all positive sera collected at chronic stages of the disease, findings that correlate with earlier studies using LppB [14]. Early diagnosis and treatment of CBPP including the ability to detect chronic stages of the disease is important since it could accelerate CBPP control and/or eradication programs by testing, isolating, and/or treating infected cattle. Treatment prevents transmission of disease from carriers to naïve animals. Macrolides such as danofloxacin and more recently tulathromycin have been reported to reduce the spread of CBPP to healthy in-contact cattle that are treated at an appropriate time [27, 28].

Ten antigens (Table 2) were used to screen sera from the control group, subunit vaccinated, and CBPPinfected cattle. Amongst the ten antigens used in an iELISA platform in this study, MSC_0499, MSC_0776, MSC_0636, and LppB were determined as the most sensitive and specific antigens for a DIVA test. MSC_0499 


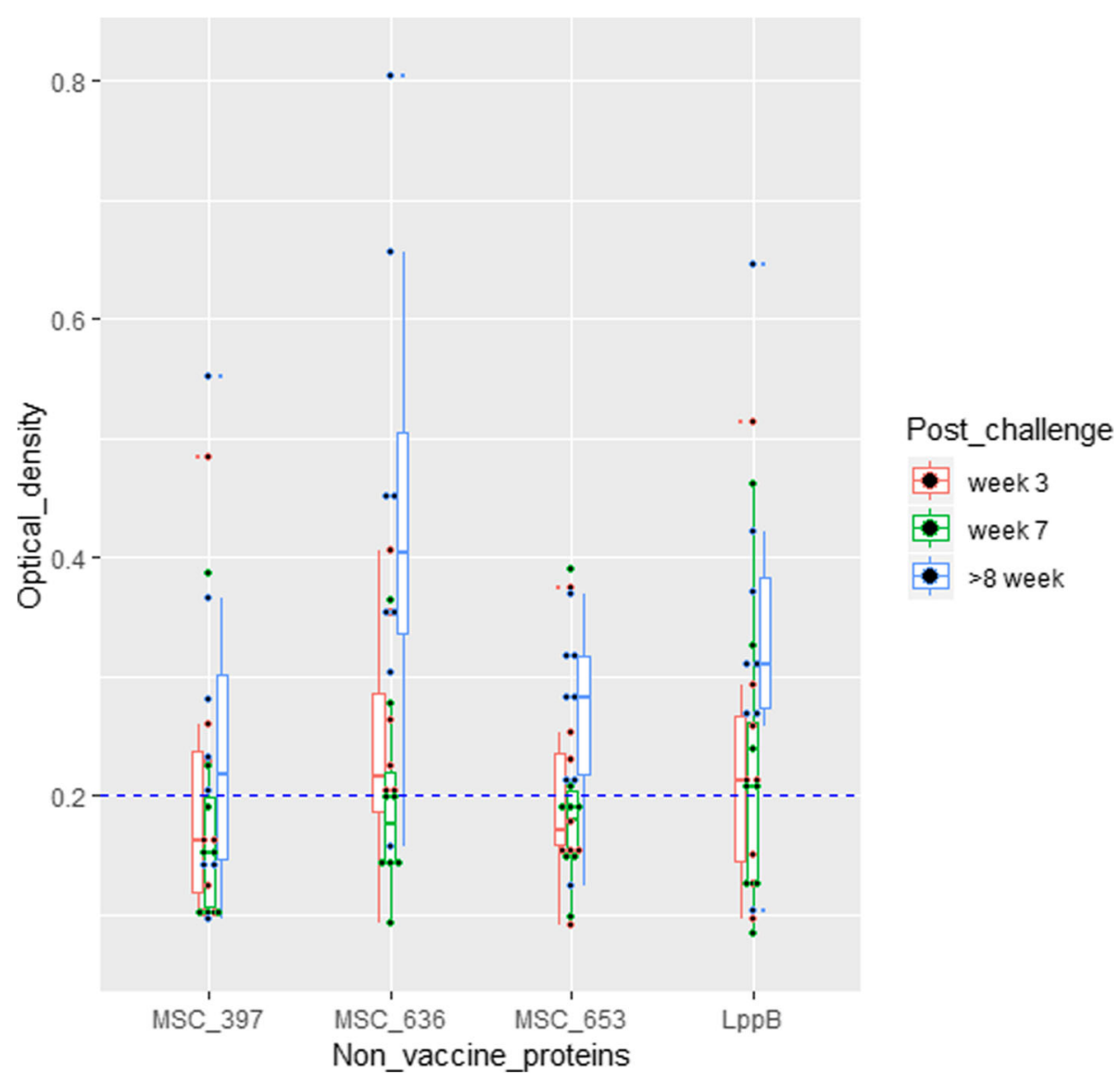

Fig. 5 Box and scatter plots showing ODs of non-vaccine antigens screened against sera from CBPP-infected cattle. MSC_0636 significantly detected antibodies in sera from infected cattle followed by LppB. As expected, the highest seroconversion of animals was observed in the chronic disease stage (blue boxes) at an OD cut-off 0.2

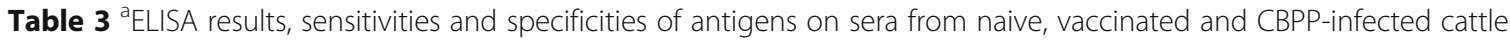

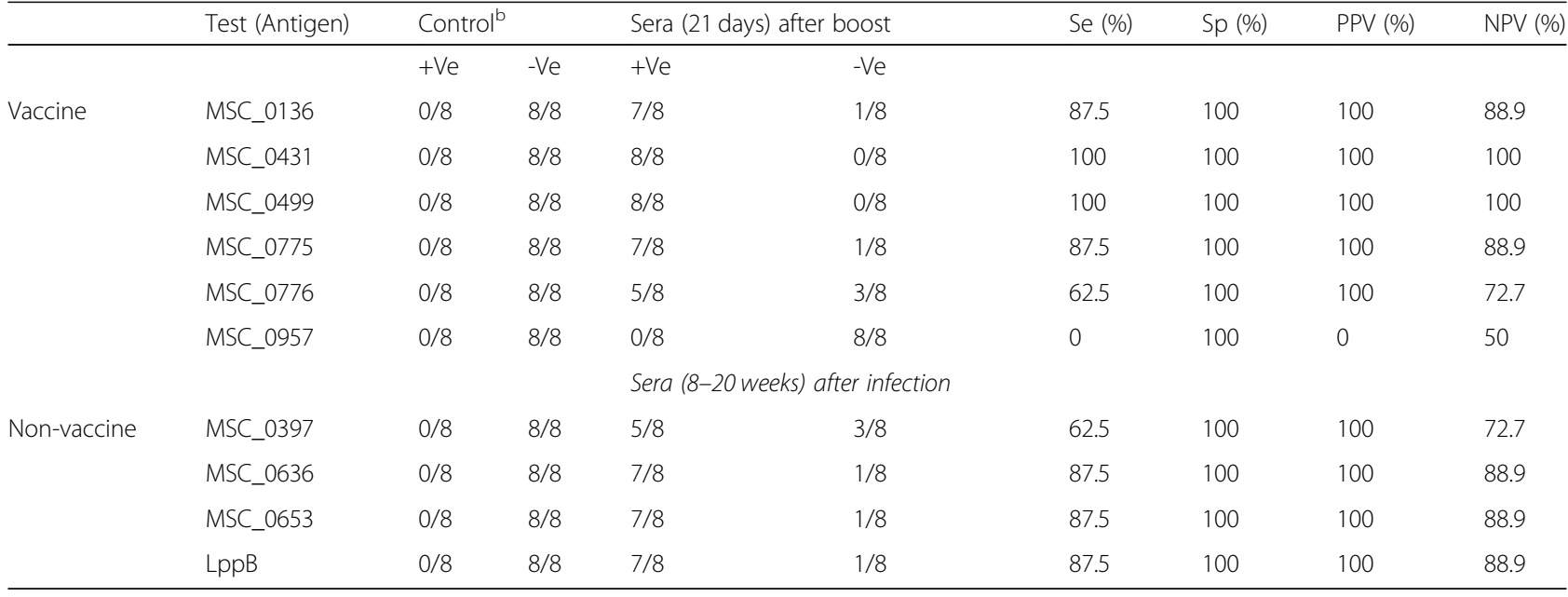

${ }^{a}$ This table represents results through the use of sera from naïve, CBPP-infected, and subunit-vaccinated cattle only. +Ve., positive; -Ve., negative. Cohen's kappa index value, classified as very good (1-0.76), good (0.75-0.61), acceptable $(0.6-0.4)$, and poor $(<0.4)$

${ }^{\mathrm{b}}$ All CBPP-negative serum samples obtained from a group of 8 cattle $(n=8)$ were correctly identified as negative 
and MSC_0776 were selected as the best performing vaccine proteins because the antigens detected vaccinated animals and showed very little reactivity with sera from infected animals, while MSC_0636 and LppB were selected as best performing non-vaccine proteins since the antigens detected infected animals and showed very little reactivity with sera from vaccinated animals. Our data suggest that MSC_0499 and MSC_0636 are the most sensitive and specific antigens for the development of serological assays for diagnosis and DIVA tests for CBPP.

\section{Conclusion}

This study characterized ten $\mathrm{Mmm}$ antigens for use in a potential novel DIVA diagnostic for CBPP. The MSC_ 0499, MSC_0776, MSC_0636, and LppB proteins were able to differentiate cattle vaccinated with the subunit vaccine from those infected with a virulent $\mathrm{Mmm}$ Afade strain. Our preliminary analysis shows that $\mathrm{Mmm}$ antigens are potential targets for developing a DIVA diagnostic assay, though further testing of field sera from vaccinated and infected animals collected at different time intervals, and a cocktail made of the four antigens, should be undertaken to establish how useful a diagnostic test based on $\mathrm{Mmm}$ antigens will be.

\section{Methods}

All protocols of this study were designed and performed in strict accordance as per applicable animal welfare regulations with the approval of KALRO-VSRI, Institutional Animal Care and Use Committee (IACUC): VSRI/ IACUC009/15072016. Cattle owners were informed about the study before the purchase of cattle. There was no requirement for Vaccine and Infectious Disease Organization-International Vaccine Centre (VIDO-InterVac) to obtain ethical approval in Canada since the trials were conducted in Kenya.

\section{Sera from naive and CBPP infected cattle}

Sera were obtained from the experiments described previously [29]. Thirty two serum samples were used in this study. Twenty-four of the sera were collected from eight male Zebu cattle ( 2 to 4 years old, weighing $105-214 \mathrm{~kg}$ ) that had been infected with $\mathrm{Mmm}$ Afade and collected at different clinical stages: acute (3 weeks), subacute (7 weeks), and chronic (above 8 weeks) of the disease. From these eight infected animals at necropsy time, three had chronic sequestrae and the other five had consolidating acute lesions. $\mathrm{Mmm}$ was isolated from lung specimens of all the 8 CBPP-infected animals. The remaining serum samples were collected from eight male naïve zebu cattle ( 2 to 4 years old, weighing $105-214 \mathrm{~kg}$ ). The sera from naïve cattle (sourced from Kakamega county, a CBPP negative area in Kenya) were well characterized and confirmed to be negative by both OIE prescribed serological tests, a CFT, and a c-ELISA.

\section{Sera from control group and subunit vaccinated cattle} Male Boran cattle (1 to 2 years old, weighing 249-390 $\mathrm{kg}$ ) purchased from a CBPP free ranch, Kapiti Plains Estate Kenya were used in this study as previously described [7]. Briefly, using Microsoft Excel's function, cattle were randomly assigned into three groups comprising 24 animals (Table 1). Eight of the animals from the unvaccinated group, were used as controls, while sixteen cattle were vaccinated with two test vaccines, each group consisting of eight animals (Table 1). All experimenters did not know the correspondence between the groups and treatments before the end of the trial and the blinding integrity was maintained throughout the study period. The vaccines used in this study (Table 1) were formulated at the VIDO-InterVac. Animals in each group were restrained in a cage, vaccinated twice subcutaneously using an 18-gauge needle with $2 \mathrm{ml}$ of the vaccine formulation (Table 1), first on the left side of the neck on day 0 , and the booster vaccine on the right side of the neck on day 28. Sampling was done on the following days: $0,28,49,60,81,102$, and 123 . The blood samples were obtained from the jugular vein and collected into labeled BD Vacutainer tubes (Becton, Dickson \& Company, USA), then allowed to coagulate at room temperature for $2 \mathrm{~h}$. The coagulated blood was centrifuged to separate the serum which was then aliquoted into Nunc ${ }^{\circ}$ CryoTubes $^{\bullet}$ (Sigma-Aldrich ${ }^{\circ}$, Germany). The serum samples were transported from the site of the trial (KALRO-VSRI) to Biosciences eastern and central Africa (BecA-ILRI) and stored at $-20^{\circ} \mathrm{C}$ until further serological analysis to develop a DIVA test. Table 1 shows details of trial vaccines and controls. At 6 weeks postchallenge, cattle were euthanized by stunning with a captive bolt pistol and exsanguination. The carcasses were opened and lungs examined for CBPP lesions. No study animal entered the human food chain. The carcasses of all animals were disposed of in deep lime pits.

\section{Expression and purification of proteins for indirect ELISA}

The genome sequences of non-vaccine antigens (MSC0397, MSC_0636, and MSC-0653) were sent to GenScript USA Inc. for cloning into the pQE60 expression vector after which they were transformed into competent $E$. coli cells (BL 21 DE3 STAR). The strains containing these plasmids were kept at $-80^{\circ} \mathrm{C}$ until use. The gene encoding LppB was cloned into pETite C-His Kan expression vector, transformed, and sent in glycerol stocks to BecA-ILRI from the Agricultural Research Council (ARC)-Onderstepoort Veterinary Institute (OVI), South Africa for expression and purification of antigens. The strains containing plasmids encoding the 
vaccine antigens (MSC_0136, MSC_0431, MSC_0499, and MSC_0775) were provided by the VIDO-InterVac, University of Saskatchewan, Canada. The agar slants were immediately streaked on agar plates containing $100 \mu \mathrm{g} / \mathrm{ml}$ ampicillin, incubated overnight, glycerol stocks prepared, and stored for future use. VIDO-InterVac also provided the MSC_0776 and MSC_0957 purified antigens.

The recombinant $\mathrm{Mmm}$ proteins were expressed as described with minor modifications [4]. Briefly, $250 \mathrm{ml}$ of broth containing $100 \mu \mathrm{g} / \mathrm{ml}$ ampicillin was inoculated with $5 \mathrm{ml}$ of the overnight culture in a 1-l flask and incubated at $37^{\circ} \mathrm{C}$ in a shaker incubator at $220 \mathrm{rpm}$. The expression of proteins was induced in exponentially growing bacterial cultures (absorbance at $600 \mathrm{~nm}, 0.5-0.6$ ) by the addition of $1 \mathrm{mM}$ IPTG followed by incubation for $6 \mathrm{~h}$ at $37^{\circ} \mathrm{C}$ at $220 \mathrm{rpm}$. The culture was then centrifuged in Beckman coulter Avanti J-301 at $8000 \mathrm{rpm}$ for $15 \mathrm{~min}$. The supernatant was discarded and pellets suspended in $1 \mathrm{ml}$ of $25 \%$ sucrose $/ 50 \mathrm{mM}$ tris $\mathrm{pH} 8.0$, transferred to $50 \mathrm{ml}$ Corning tubes, and stored at $-80^{\circ} \mathrm{C}$ until the day of purification. The LppB protein was expressed as earlier described [24].

The cell pellets were thawed for $15 \mathrm{~min}$ on ice and suspended in lysis buffer (with $8 \mathrm{M}$ urea) at $10 \mathrm{ml}$ per gram wet weight. $0.1 \mathrm{mM}$ Phenyl methyl sulfonyl fluoride (PMSF)-Sigma Aldrich, USA was added to inhibit proteases. The suspended pellet was mixed for $60 \mathrm{~min}$ at room temperature (RT) taking care to avoid foaming. Recombinant proteins were purified by affinity chromatography on Nickel resin. The lysate was first centrifuged at 10, 000x g for $30 \mathrm{~min}$ at $15^{\circ} \mathrm{C}$ to pellet the cellular debris and supernatant saved. $1 \mathrm{ml}$ of a 50\% Ni-NTA slurry (Sigma Aldrich, USA) was added to $10 \mathrm{ml}$ lysate and mixed gently by shaking for $30 \mathrm{~min}$ at RT. The lysate-resin mixture was loaded into a column (BioRad, USA) and the flow-through collected for SDS-PAGE analysis. The columns were washed three times with $4 \mathrm{ml}$ of wash buffer at $\mathrm{pH} 6.3$ and wash fractions kept for SDS-PAGE analysis. Recombinant proteins were eluted three times with $1 \mathrm{ml}$ elution buffer containing; $8 \mathrm{M}$ urea, $0.1 \mathrm{M} \mathrm{NaH}_{2} \mathrm{PO}_{4}, 1.5 \mathrm{M} \mathrm{NaCl}, 0.125 \mathrm{M}$ imidazole at $\mathrm{pH}$ 7.4.

The purified proteins were extensively dialyzed in $4 \mathrm{M}$ urea for $1.5 \mathrm{~h}$ then PBS for either $1 \mathrm{~h}$ (MSC_0499, MSC 0636, MSC_0653, and MSC_0775) or overnight at $4^{0} \bar{C}$ (MSC_0136, MSC_0397, MSC_0431, and LppB) and concentrated using polyethylene glycol from Sigma Aldrich, Germany. Quantification was performed as described by the BCA Protein Assay Kit (Thermo Scientific, USA). Antigens $(5 \mu \mathrm{l}$ of each) were mixed with $2 \mu \mathrm{l} 2 \mathrm{x}$ SDS sample buffer, and resolved in 10\% SDS-PAGE gel.

\section{Characterization of the cattle immune responses by indirect ELISA}

The protocol was adapted from Nkando et al., [7] as follows: 96-well Nunc-Immuno-plate MaxiSorp; Thermo
Fisher Scientific, USA were coated with $100 \mu \mathrm{l} /$ well-containing $1 \mu \mathrm{g} / \mathrm{ml}$ of antigen in $\mathrm{Na}_{2} \mathrm{CO}_{3}: \mathrm{NaHCO}_{3}$ buffer (3.03 $\mathrm{g} \mathrm{Na}_{2} \mathrm{CO}_{3} ; 6.0 \mathrm{~g} \mathrm{NaHCO}_{3}$ in1000 ml dH $\mathrm{d}_{2} \mathrm{O}$ for 100 $\mathrm{mM}$ at $\mathrm{pH}$ 9.6, diluted to $1: 10$ with $\mathrm{dH}_{2} \mathrm{O}$ before use) and incubated at $4^{0} \mathrm{C}$ overnight. The following day, plates were washed five times with $300 \mu$ l wash buffer \{PBS with $0.05 \%$ Tween 20 (PBST) \} followed by blocking with $200 \mu$ l blocking buffer (PBST $+0.5 \%$ Horse serum from Gibco Life Technologies ${ }^{\mathrm{Tw}}$, New Zealand) for $1 \mathrm{~h}$ in a shaker incubator at $37^{\circ} \mathrm{C}$. The wells were washed five times with $300 \mu \mathrm{l}$ wash buffer before adding $100 \mu \mathrm{l}$ serum samples (diluted 4-fold 1:100 in row A to 1: 409, 600 in row G) followed by $1 \mathrm{~h}$ incubation at $37^{\circ} \mathrm{C}$ and five washes as described above. In total, $100 \mu \mathrm{l}$ alkaline phosphatase-conjugated rabbit-anti-bovine IgG antibody (KPL151-12-06, Sera Care, USA), diluted 1: 5000 in PBST, was added to each well before incubation for $1 \mathrm{~h}$ at $37^{\circ} \mathrm{C}$. Following five washes as above, $100 \mu \mathrm{l} /$ well of para-Nitro phenyl phosphate (pNPP substrate, Sigma Aldrich, USA) was added. The reaction mixture was incubated for $45 \mathrm{~min}$ in the dark and the absorbance (OD) measured at $405 \mathrm{~nm}$ in a BioTek Synergy HT, USA plate reader.

\section{Determination of cut-off point, diagnostic sensitivity, and specificity of $\mathrm{Mmm}$ antigens}

The iELISAs were done in duplicate and repeated on three different occasions. The negative and positive control sera were placed in duplicates in the first and last row of plates coated with $1 \mu \mathrm{g} / \mathrm{ml}$ of each antigen. No serum sample was placed in the second row and an OD of this row was subtracted from the OD containing sera. To analyze iELISA data based on $\mathrm{Mmm}$ antigens, the change-point method was determined as the cut-off point formula of a mean +3 standard deviation of negative controls [30]. Percentage positivity $(\mathrm{PP})$ was calculated as follows: $\mathrm{PP}=$ $\{(\mathrm{ODsample}-\mathrm{OD}-\mathrm{ve}$ control)/ (OD + ve control-OD-ve control) $\} \times 100 \%$ [31]. The standardized data was plotted in the box and scatter plots and cut-off OD values were used to discriminate the naïve, CBPP-infected, and subunit vaccinated animals. Sensitivity (Se), specificity (Sp), positive predictive value (PPV), and negative predictive value $(\mathrm{NPV})$ were calculated as follows; Se: $\{\mathrm{TP} /(\mathrm{TP}+$ $\mathrm{FN})\} \times 100 \%$, where $\mathrm{TP}=$ true positive, $\mathrm{FN}=$ false negative; Sp: $\{\mathrm{TN} /(\mathrm{TN}+\mathrm{FP})\} \times 100 \%$, where $\mathrm{TN}=$ true negative, $\mathrm{FP}=$ false positive; $\mathrm{PPV}:\{\mathrm{TP} /(\mathrm{TP}+\mathrm{FP})\} \times 100 \%$; NPV: $\{\mathrm{TN} /(\mathrm{TN}+\mathrm{FN})\} \times 100 \%[8,32]$. Sera from naïve and subunit vaccinated cattle were used to determine Se and Sp of $\mathrm{Mmm}$ vaccine antigens, while sera from naive and CBPP infected cattle were used to determine Se and Sp of $M m m$ non-vaccine antigens. In this study, the number of animals per group $(n=8)$ was based on previous immunization trials [7,33-36]. The resource equation method was used to show that our sample size was 
adequately powered to address the research question. Briefly, a value " $E "$ " Total number of animals - Total number of groups; whereas in our study the "E" value for naïve group and CBPP-infected or control group and subunit-vaccinated cattle was determined as follows: $\{(8 \times$ 2) $-2=14\}$. Our "E" value of 14 lied between 10 and 20 , which is the acceptable limit [37, 38].

\section{Data analysis}

Data for iELISA with different antigens were entered into MS Excel (Microsoft ${ }^{\circ}$ Excel, Washington, 2016) and transferred into SPSS version 22, analyzed by two-way Analysis of Variance (ANOVA) and least significance difference used to separate the means. Data were imported into R software, version 3.6.0 (R Core Team, 2019) for analysis and drawing of the box and scatter plots. Cohen's Kappa measurement assessed the level of agreement between the $\mathrm{Mmm}$ antigens.

\section{Supplementary information}

Supplementary information accompanies this paper at https://doi.org/10. 1186/s12917-020-02453-w.

Additional file 1. A comparison of the performance of vaccine and non-vaccine antigens. The SPSS was used to compare the performance of vaccine and non-vaccine antigens on sera from the control/naïve group, CBPP-infected, and subunit-vaccinated cattle.

Additional file 2. A comparison of the non-vaccine antigens on CBPP clinical stages. The SPSS was used to compare the performance of nonvaccine antigens on sera (collected at different clinical stages) from the naïve group and CBPP-infected cattle.

Additional file 3. ODs returned after testing sera from the control group and subunit-vaccinated cattle. The ODs were used to draw box and scatter plots, and determine the sensitivity and specificity of $\mathrm{Mmm}$ vaccine antigens.

Additional file 4. ODs returned after testing sera from naïve group and CBPP-infected cattle. The ODs were used to draw the box and scatter plots.

Additional file 5. ODs used to calculate the sensitivity and specificity of the naive group and CBPP-infected cattle. The ODs were used to determine the sensitivity and specificity of $\mathrm{Mmm}$ non-vaccine antigens.

\section{Acknowledgments}

Hezron Wesonga of KALRO-VSRI provided sera from control groups, subunit vaccinated, and CBPP-infected cattle. Daniel Nthiwa assisted in data analysis. Dr. Phil Toye and Dr. Paul Hodgson edited the English in the paper.

\section{Authors' contributions}

$H O L, J N, J P, A P, V G$, and EB contributed to the conception and design of the study. HOL, AK, and MO performed the animal experiments at VSRI. HOL was responsible for the laboratory work at BecA-ILRI. TP, JP, EB were responsible for the preparation of Mmm plasmids and/or antigens (MSC_0136, MSC_0431, MSC_0499, MSC_0775, MSC_0776, and MSC_0957) at VIDOInterVac, Canada and shipping to KALRO-VSRI. AM was responsible for the cloning of the plasmid encoding the Mmm LppB gene at OVI, South Africa, and shipping to BecA-ILRI. AP and VG were principal investigators for CBPP subunit vaccines. MK and RP offered technical support during laboratory work at BecA-ILRI. HOL, JN, and DO wrote the paper and also contributed to the interpretation of the data. All authors read and approved the manuscript for submission.

\section{Funding}

$\mathrm{HOL}, \mathrm{JN}, \mathrm{JP}, \mathrm{AP}, \mathrm{VG}, \mathrm{EB}$, and TP were funded by the Canadian International Food Security Research Fund (CIFSRF) grant 107849 of the International Development Research Centre (IDRC) that supported on-station trials on safety and efficacy of subunit vaccines. HOL was also sponsored by VACN ADA grant DCI-FOOD/2009/226-469 and the BecA-ILRI Hub through the African Biosciences Challenge Fund (ABCF) Program. The ABCF program is funded by the Australian Department for Foreign Affairs and Trade (DFAT) through the BecA-CSIRO partnership; the Syngenta Foundation for Sustainable Agriculture (SFSA); the Bill \& Melinda Gates Foundation (BMGF); the UK Department for International Development (DFID) and the Swedish International Development Cooperation Agency (Sida). The VACNADA grant determined a suitable model of inoculation of animals during vaccine safety and efficacy studies while the ABCF grant supported the laboratory work of this study. The findings and conclusions contained within this paper are those of the authors and do not necessarily reflect positions or policies of the CIFSRF or VACNADA or ABCF grants. The funders (CIFSRF, VACNADA and ABCF grants) had no role in study design, data collection and analysis, decision to publish, or preparation of the manuscript.

\section{Availability of data and materials}

The data on CBPP DIVA assay are the data used or analyzed in this paper and are available from the corresponding author (Harrison O. Lutta) on reasonable request. The datasets for CBPP subunit vaccines (International Patent Application No. PCT/CA2016/050864) are available on reasonable request from Dr. Andrew Potter, VIDO-InterVac.

\section{Ethics approval and consent to participate}

This study involved cattle experiments only. The study protocol was submitted to and approved by the Animal Care and Use Committee of the KALRO-VSRI/ Approval Number: VSRI/IACUC009/15072016.

\section{Consent for publication}

Not applicable. The paper does not contain any data from specific persons.

\section{Competing interests}

The authors declare that the research was conducted in the absence of any commercial or financial relationships that could be construed as a potential conflict of interest. The authors declare that they have no competing interests.

\section{Author details}

${ }^{1}$ Kenya Agricultural and Livestock Research Organization, Biotechnology Research Institute, P.O. Box 14733-00800, Nairobi, Kenya. ${ }^{2}$ School of Biological Sciences, University of Nairobi, P.O. Box 30197-00100, Nairobi, Kenya. ${ }^{3}$ Agricultural Research Council - Onderstepoort Veterinary Research, Private Bag X5, Onderstepoort, Pretoria 0110, South Africa. ${ }^{4}$ Vaccine and Infectious Disease Organization-International Vaccine Centre (VIDO-InterVac), University of Saskatchewan, 120 Veterinary Rd, Saskatoon, SK S7N 5E3, Canada. ${ }^{5}$ Biosciences eastern and central Africa-International Livestock Research Institute, Old Naivasha Road, P.O. Box 30709-00100, Nairobi, Kenya. ${ }^{6}$ Kenya 
Agricultural and Livestock Research Organization, Veterinary Science Research Institute, P.O. Box 32-00902, Kikuyu, Kenya.

\section{Received: 27 February 2020 Accepted: 2 July 2020 Published online: 10 July 2020}

\section{References}

1. Manso-Silvan L, Vilei EM, Sachse K, Djordjevic SP, Thiaucourt F, Frey J. Mycoplasma leachii sp. nov. as a new species designation for Mycoplasma sp. bovine group 7 of Leach, and reclassification of Mycoplasma mycoides subsp. mycoides Large Colony as a serovar of Mycoplasma mycoides subsp. capri. Int J Syst Evol Microbiol. 2009;59:1353-8.

2. Sacchini F, Naessens J, Awino E, Heller M, Hlinak A, Haider W, Sterner-Kock A, Jores J. A minor role of CD4+ T lymphocytes in the control of a primary infection of cattle with Mycoplasma mycoides subsp. mycoides. Vet Res. 2011:42:77.

3. Tambi NE, Maina WO, Ndi C. An estimation of the economic impact of contagious bovine pleuropneumonia in Africa. Rev Sci Technol Office Int Epizootics. 2006;25:999-1011.

4. Perez-Casal J, Prysliak T, Maina T, Wang Y, Townsend H, Berberov E, Nkando I, Wesonga H, Liljander A, Jores J, Naessens J, Gerdts V, Potter A. Analysis of immune responses to recombinant proteins from strains of Mycoplasma mycoides subsp. mycoides, the causative agent of contagious bovine pleuropneumonia. Vet Immunol Immunopathol. 2015;168:103-10.

5. Wise KS, Calcutt MJ, Foecking MF, Madupu R, DeBoy RT, Roske K, Hvinden ML, Martin TR, Durkin AS, Glass JI, Methe BA. Complete genome sequences of Mycoplasma leachii strain PG50T and the pathogenic Mycoplasma mycoides subsp. mycoides small colony biotype strain Gladysdale. J Bacteriol. 2012;194:4448-9.

6. Westberg J, Persson A, Holmberg A, Goesmann A, Lundeberg J, Johansson KE, Pettersson B, Uhlen M. The genome sequence of Mycoplasma mycoides subsp. mycoides SC type strain PG1, the causative agent of contagious bovine pleuropneumonia (CBPP). Genome Res. 2004;14:221-7.

7. Nkando I, Perez-Casal J, Mwirigi M, Prysliak T, Townsend H, Berberov E, Kuria J, Mugambi J, Soi R, Liljander A, Jores J, Gerdts V, Potter A, Naessens J, Wesonga $\mathrm{H}$. Recombinant Mycoplasma mycoides proteins elicit protective immune responses against contagious bovine pleuropneumonia. Vet Immunol Immunopathol. 2016;171:103-14.

8. Heller M, Gicheru N, Tjipura-Zaire G, Muriuki C, Yu M, Botelho A, Naessens J, Jores J, Liljander A. Development of a novel cocktail enzyme-linked Immunosorbent assay and a field-applicable lateral-flow rapid test for diagnosis of contagious bovine Pleuropneumonia. J Clin Microbiol. 2016;54:6.

9. Hamsten C, Westberg J, Bolske G, Ayling R, Uhlen M, Persson A. Expression and immunogenicity of six putative variable surface proteins in Mycoplasma mycoides subsp. mycoides SC. Microbiology. 2008;154:539-49.

10. Hamsten C. Protein-based approaches to understand and prevent contagious bovine pleuropneumonia. A thesis submitted to the School of Biotechnology. Stockholm: Royal Institute of Technology (KTH); 2009.

11. Neiman M, Hamsten C, Schwenk MJ, Bolske G, Persson A. Multiplex screening of surface proteins from Mycoplasma mycoides subsp. mycoides small Colony for an antigen cocktail enzyme-linked Immunosorbent assay. Clin Vaccine Immunol. 2009:16:1665-74.

12. Naseem S, Meens J, Jores J, Heller M, Dubel S, Hust M, Gerlach GF. Phage display-based identification and potential diagnostic application of novel antigens from Mycoplasma mycoides subsp. mycoides small colony type. Vet Microbiol. 2010;142:285-92.

13. Fischer A, Santana-Cruz I, Hegerman J, Gourle H, Schieck E, Lambert M, Nadendla S, Wesonga H, Miller RA, Vashee S, Weber J, Meens J, Frey J, Jores J. High-quality draft genomes of the Mycoplasma mycoides subsp. mycoides challenge strains Afadé and B237. Stand Genomic Sci. 2015;10:89.

14. Lutta OH, Mather A, Maina WT, Odongo D, Ndiwa NN, Wesonga $\mathrm{OH}$ Naessens J. Preliminary findings of lipoprotein B in detecting cattle chronically infected with CBPP. J Vet Sci Med Diagnosis. 2018;7:2.

15. Han X, Khan FA, Zhu X, Zhang R, Mustafa R, Hu C, Chen Y, Chen H, Guo A Establishment of an antibody avidity test to differentiate vaccinated cattle from those naturally infected with Mycoplasma bovis. Vet J. 2015;203:79-84

16. Khan AF, Zhao G, Guo Y, Faisal M, Chao J, Chen X, He C, Menghwar H, Dad R, Zubair M, Hu C, Chen Y, Chen H, Rui Z, Guo A. Proteomics identification and characterization of MbovP730 as a potential DIVA antigen of Mycoplasma bovis. Oncotarget. 2018;9(47):28322-36.
17. Zhang R, Han X, Chen Y, Mustafa R, Qi J, Chen X, Hu C, Chen H, Guo A. Attenuated Mycoplasma bovis strains protect against virulent infection in calves. Vaccine. 2014;32:3107-14.

18. Janis C, Bischof D, Gourgues G, Frey J, Blanchard A, Sirand-Pugnet P. Unmarked insertional mutagenesis in the bovine pathogen Mycoplasma mycoides subsp mycoides SC: characterization of an LppQ mutant. Microbiology. 2008;154:2427-36.

19. Abdo EM, Nicolet J, Frey J. Antigenic and genetic characterization of lipoprotein LppQ from Mycoplasma mycoides subsp. mycoides small Colony. Clin Diagnosis Lab Immunol. 2000;7:588-95.

20. Dedieu L, Totte P, Rodrigues V, Vilei EM, Frey J. Comparative analysis of four lipoproteins from Mycoplasma mycoides subsp. mycoides small Colony identifies LppA as a major T-cell antigen. Comparative Immunology. Microbiol Infect Dis. 2009;33:279-90.

21. Bruderer U, Regalla J, Abdo E, Huebschle OJ, Frey J. Serodiagnosis and monitoring of contagious bovine Pleuropneumonia (CBPP) with an indirect ELISA based on the specific lipoprotein Q of Mycoplasma mycoides subsp. mycoides SC. Vet Microbiol. 2002;84:195-205.

22. Vilei ME, Frey M. Detection of Mycoplasma mycoides subsp. mycoides SC in broncho-alveolar lavage fluids of cows based on a TaqMan real-time PCR discriminating wild type strains from an LppQ-mutant vaccine strain used for DIVA-strategies. J Microbiol Methods. 2010;81:211-8.

23. Dedieu-Engelmann L. Contagious bovine pleuropneumonia: a rationale for the development of a mucosal sub-unit vaccine. Comp Immunol Microbiol Infect Dis. 2008;31:227-38.

24. Miltiadou DR, Mather A, Vilei EM, DHD P. Identification of genes coding for B cell antigens of Mycoplasma mycoides subsp. mycoides Small Colony (MmmSC) by using a phage display. BMC Microbiol. 2009;9:215.

25. Dedieu L, Breard A, Le-Goff C, Lefevre PC. Diagnosis of contagious bovine pleuropneumonia: problems and new developments. Rev Sci Technol Office Int Epizootics. 1996;15:1331-53.

26. Sidibé $C A$, Grosbois $V$, Thiaucourt F, Niang M, Lesnoff M, Roger F. Performance evaluation of two serological tests for CBPP detection in an enzootic area using a Bayesian framework. Trop Anim Health Prod. 2012; 44(6):1233-8.

27. Huebschle O, Ayling R, Godinho K, Lukhele O, Tjipura-Zaire G, Rowan T, Nicholas R. Danofloxacin (AdvocinTM) reduces the spread of contagious bovine pleuropneumonia to healthy in-contact cattle. Res Vet Sci. 2006;81: 304-9.

28. Muuka G, Otina B, Wesonga H, Bowa B, Gicheru N, Stuke K, Poole J, Salt J, Colston A. Evaluation of new generation macrolides for the treatment and metaphylaxis of contagious bovine pleuropneumonia in cattle experimentally infected with Mycoplasma mycoides subsp mycoides. BMC Vet Res. 2019;15:451.

29. Lutta $\mathrm{OH}$, Wesonga $\mathrm{OH}$, Odongo $\mathrm{D}$, Thiaucourt F, Naessens J. Inoculation of Mycoplasma mycoides mycoides by endotracheal intubation produces a milder disease than by contact transmission. Bull Animal Health Prod Afr. 2017:65(3):481-9.

30. Lardeux F, Torrico G, Aliaga C. Calculation of the ELISA's cut-off based on the change-point analysis method for detection of Trypanosoma cruzi infection in Bolivian dogs in the absence of controls. Mem Inst Oswaldo Cruz. 2016;111(8):501-4.

31. Le Goff C, Thiaucourt F. A competitive ELISA for the specific diagnosis of contagious bovine pleuropneumonia (CBPP). Vet Microbiol. 1998;60:179-91.

32. Amanfu W, Sediadie S, Masupu KV, Raborokgwe V, Benkirane A, Geiger R, Thiaucourt F. Comparison between c-ELISA and CFT in detecting antibodies to Mycoplasma mycoides subsp mycoides biotype SC in cattle affected by CBPP in Botswana. Ann New York Acad Sci. 2000;916:364-9.

33. Prysliak T, Maina T, Yu L, Suleman M, Jimbo S, Perez-Casal J. Induction of a balanced lgG1/lgG2 immune response to an experimental challenge with Mycoplasma bovis antigens following a vaccine composed of Emulsigen ${ }^{\text {TM }}$, IDR peptide 1002, and poly I:C. Vaccine. 2017;35:6604-10.

34. Prysliak T, Maina T, Perez-Casal J. Th-17 cell mediated immune responses to Mycoplasma bovis proteins formulated with Montanide ISA61 VG and curdlan are not sufficient for protection against an experimental challenge with Mycoplasma bovis. Vet Immunol Immunopathol. 2018;197:7-14.

35. Mwirigi M, Nkando I, Aye R, Soi R, Ochanda H, Berberov E, Potter A, Gerdts $\checkmark$, Perez-Casal J, Naessens J, Wesonga H. Experimental evaluation of inactivated and live attenuated vaccines against Mycoplasma mycoides subsp. mycoides. Vet Immunol Immunopathol. 2016;169:63-7.

36. Mwirigi M, Nkando I, Olum M, Attah-Poku S, Ochanda H, Berberov E, Potter A, Gerdts V, Perez-Casal J, Wesonga H, Soi R, Naessens J. Capsular 
polysaccharide from Mycoplasma mycoides subsp. mycoides shows potential for protection against contagious bovine pleuropneumonia. Vet Immunol Immunopathol. 2016;178:64-9.

37. Festing MF, Altman DG. Guidelines for the design and statistical analysis of experiments using laboratory animals. J Institute Lab Animal Res. 2002;43: 244-58.

38. Festing MF. Design and statistical methods in studies using animal models of development. J Institute Lab Animal Res. 2006:47:5-14.

\section{Publisher's Note}

Springer Nature remains neutral with regard to jurisdictional claims in published maps and institutional affiliations.

Ready to submit your research? Choose BMC and benefit from:

- fast, convenient online submission

- thorough peer review by experienced researchers in your field

- rapid publication on acceptance

- support for research data, including large and complex data types

- gold Open Access which fosters wider collaboration and increased citations

- maximum visibility for your research: over $100 \mathrm{M}$ website views per year

At BMC, research is always in progress.

Learn more biomedcentral.com/submissions 